\title{
Regularizing and decay rate estimates for solutions to the Cauchy problem of the Debye-Hückel system
}

\author{
Jihong Zhao, Qiao Liu, and Shangbin Cui
}

\begin{abstract}
In this paper we establish some regularizing and decay rate estimates for mild solutions of the Debye-Hückel system. We prove that if the initial data belong to the critical Lebesgue space $L^{\frac{n}{2}}\left(\mathbb{R}^{n}\right)$, then the $L^{q}$-norm $\left(\frac{n}{2} \leq q \leq \infty\right)$ of the $\beta$ th order spatial derivative of mild solutions are majorized by $K_{1}\left(K_{2}|\beta|\right)^{|\beta|} t^{-\frac{|\beta|}{2}-1+\frac{n}{2 q}}$ for some constants $K_{1}$ and $K_{2}$. These estimates particularly imply that mild solutions are analytic in the space variable, and provide decay estimates in the time variable for higher-order derivatives of mild solutions. We also prove that similar estimates also hold for mild solutions whose initial data belong to the critical homogeneous Besov space $\dot{B}_{p, \infty}^{-2+\frac{n}{p}}\left(\mathbb{R}^{n}\right)\left(\frac{n}{2}<p<n\right)$.

Mathematics Subject Classification (2010). 35B65, 35K45, 35K55.
\end{abstract}

Keywords. Debye-Hückel system, Cauchy problem, Mild solution, Regularizing estimate, Decay rate estimate.

\section{Introduction}

In this paper we consider the Cauchy problem of the Debye-Hückel system:

$$
\begin{aligned}
& \partial_{t} v=\nabla \cdot(\nabla v-v \nabla \phi) \quad \text { in } \mathbb{R}^{n} \times(0, \infty), \\
& \partial_{t} w=\nabla \cdot(\nabla w+w \nabla \phi) \quad \text { in } \mathbb{R}^{n} \times(0, \infty), \\
& \Delta \phi=v-w \quad \text { in } \mathbb{R}^{n} \times(0, \infty), \\
& v(x, 0)=v_{0}(x), \quad w(x, 0)=w_{0}(x) \quad \text { in } \mathbb{R}^{n} .
\end{aligned}
$$

Here $v=v(x, t)$ and $w=w(x, t)$ are the scalar functions representing the densities of positive and negative ions, respectively, in an electrolyte, $\phi=\phi(x, t)$ is the scalar function representing the electric potential, and $v_{0}, w_{0}$ are the initial data of $v$ and $w$, respectively. Throughout this paper we assume that $n \geq 2$. 
The Debye-Hückel system was formulated by W. Nernst and M. Planck at the end of the nineteenth century as a basic model for the diffusion of ions in an electrolytes (cf. [5]). It also arises from mathematical modeling of semiconductors (cf. [6,20]), plasma physics (cf. [7]), chemotaxis (cf. [4]), and etc. We refer the reader to see $[1-3,6,9,11-13,17,18,21,22]$ and the references therein for previous works on this system of equations concerning existence of (large) weak solutions, (small and local) mild solutions, convergence rate estimates to stationary solutions of time-dependent solutions and other related topics.

The purpose of this paper is to establish regularizing and decay rate estimates for mild solutions of the Debye-Hückel system. Our interest on this topic is inspired by the series of interesting works of Giga and Sawada [8], Sawada [19], and Miura and Sawada [15] on the Navier-Stokes equations. In those works the authors proved the regularizing and decay rate estimates for mild solutions of the Navier-Stokes equations with initial data belonging to the usual Lebesgue space $L^{n}\left(\mathbb{R}^{n}\right)$, the homogeneous Sobolev space $\dot{H}^{\frac{n}{2}-1}\left(\mathbb{R}^{n}\right)$ and the space $B M O^{-1}$ of derivatives of $B M O$ functions, respectively (see [14] for definitions of these function spaces). It is worth noticing that these estimates not only imply that mild solutions are analytic in space variables, but also give decay rate estimates for the higher-order spatial derivatives of solutions. For the Debye-Hückel system (1.1)-(1.4), however, to the best of our knowledge, no similar research was performed before the present work. We observe that since the system (1.1)-(1.4) is different from the Navier-Stokes equations, the estimates for this system of equations are variant from those for the Navier-Stokes equations. The main new difficulty is caused by the term $\nabla \phi$ in (1.1)-(1.2), which, by (1.3), is a nonlocal functional of $v$ and $w$. To overcome this difficulty, we shall split the estimates for $\left\|\partial_{x}^{\beta} v(x)\right\|_{L^{q}}$ and $\left\|\partial_{x}^{\beta} w(x)\right\|_{L^{q}}$ into two cases: the case $\frac{n}{2} \leq q<n$ will be treated by using the classical HardyLittlewood-Sobolev inequality and the Calderón-Zygmund theorem, and the other case $n \leq q \leq \infty$ will be estimated by using the well-known GagliardoNirenberg inequality.

Before giving our main results, let us first recall the concept of mild solutions of the system (1.1)-(1.4). Note that from (1.3) we have

$$
\phi(x, t)=(-\Delta)^{-1}(w-v)(x, t)=E *(w-v)(x, t),
$$

where $E(x)=-\frac{1}{2 \pi} \log |x|$ if $n=2$ and $E(x)=\frac{1}{4} \pi^{-\frac{n}{2}} \Gamma\left(\frac{n}{2}-1\right)|x|^{-(n-2)}$ if $n \geq 3$ defined for all $x \in \mathbb{R}^{n} \backslash\{0\}$. Hence, by the well-known Duhamel principle, the system (1.1)-(1.4) can be reduced into the following integral equations:

$$
\begin{gathered}
v(t)=e^{t \Delta} v_{0}-\int_{0}^{t} e^{(t-\tau) \Delta} \nabla \cdot\left[v \nabla\left((-\Delta)^{-1}(w-v)\right)\right](\tau) d \tau \\
w(t)=e^{t \Delta} w_{0}+\int_{0}^{t} e^{(t-\tau) \Delta} \nabla \cdot\left[w \nabla\left((-\Delta)^{-1}(w-v)\right)\right](\tau) d \tau
\end{gathered}
$$

where $e^{t \Delta}$ is the convolution operator in $S^{\prime}\left(\mathbb{R}^{n}\right)$ with the heat kernel $G(x, t)=$ $(4 \pi t)^{-\frac{n}{2}} \exp \left(-\frac{|x|^{2}}{4 t}\right)$. A solution of (1.6)-(1.7) will be called a mild solution of (1.1)-(1.4). 
In what follows, for $x=\left(x_{1}, \ldots, x_{n}\right) \in \mathbb{R}^{n}$ and $\beta=\left(\beta_{1}, \ldots, \beta_{n}\right) \in \mathbb{N}_{0}^{n}$, where $\mathbb{N}_{0}=\mathbb{N} \cup\{0\}$ and $\mathbb{N}=\{1,2, \ldots\}$, we denote $\partial_{x}^{\beta}=\partial_{x_{1}}^{\beta_{1}} \cdots \partial_{x_{n}}^{\beta_{n}}$ and $|\beta|=\left|\beta_{1}\right|+\cdots+\left|\beta_{n}\right|$. Besides, for two Banach spaces $\mathcal{X}$ and $\mathcal{Y}$, the product space $\mathcal{X} \times \mathcal{Y}$ will be equipped with the norm $\|(v, w)\|_{\mathcal{X} \times \mathcal{Y}}=\|v\|_{\mathcal{X}}+\|w\|_{\mathcal{Y}}$. For simplicity, if $\mathcal{X}=\mathcal{Y}$, we simply denote $\|(v, w)\|_{\mathcal{X} \times \mathcal{X}}$ as $\|(v, w)\|_{\mathcal{X}}$. By [22], there exists $\varepsilon>0$ such that for any $\left(v_{0}, w_{0}\right) \in\left(L^{\frac{n}{2}}\left(\mathbb{R}^{n}\right)\right)^{2}$ with $\left\|\left(v_{0}, w_{0}\right)\right\|_{L^{\frac{n}{2}}} \leq \varepsilon$, the problem (1.1)-(1.4) has a unique mild solution $(v, w)$ in the product space $\left(\mathcal{X}_{p}\right)^{2}$ for any given $p \in\left(\frac{n}{2}, n\right)$, where

$\mathcal{X}_{p}=C\left([0, \infty), L^{\frac{n}{2}}\left(\mathbb{R}^{n}\right)\right) \cap\left\{u: u \in C\left((0, \infty), L^{p}\left(\mathbb{R}^{n}\right)\right)\right.$ and $\left.\sup _{t>0} t^{1-\frac{n}{2 p}}\|u\|_{L^{p}}<\infty\right\}$.

Moreover, by Karch [11], there exists $\varepsilon>0$ such that for any $\left(v_{0}, w_{0}\right) \in$ $\left(\dot{B}_{p, \infty}^{-2+\frac{n}{p}}\left(\mathbb{R}^{n}\right)\right)^{2}$ with $\left\|\left(v_{0}, w_{0}\right)\right\|_{\dot{B}_{p, \infty}^{-2+\frac{n}{p}}} \leq \varepsilon$, the problem (1.1)-(1.4) has a unique mild solution $(v, w)$ in the product space $\left(\mathcal{Y}_{p}\right)^{2}$, where

$\mathcal{Y}_{p}=C_{*}\left([0, \infty), \dot{B}_{p, \infty}^{-2+\frac{n}{p}}\left(\mathbb{R}^{n}\right)\right) \cap\left\{u: u \in C\left((0, \infty), L^{p}\left(\mathbb{R}^{n}\right)\right)\right.$ and $\left.\sup _{t>0} t^{1-\frac{n}{2 p}}\|u\|_{L^{p}}<\infty\right\}$.

Here the notation $C_{*}\left([0, \infty), \dot{B}_{p, \infty}^{-2+\frac{n}{p}}\left(\mathbb{R}^{n}\right)\right)$ denotes the set of bounded maps from $[0, \infty)$ to $\dot{B}_{p, \infty}^{-2+\frac{n}{p}}\left(\mathbb{R}^{n}\right)$ which are continuous for $t>0$ and weakly continuous for $t=0$. It is easy to see that, equipped with norms $\|v\|_{\mathcal{X}_{p}}=\sup _{t \geq 0}\|v\|_{L^{\frac{n}{2}}}+\sup _{t>0} t^{1-\frac{n}{2 p}}\|v\|_{L^{p}}$ and $\|v\|_{\mathcal{Y}_{p}}=\sup _{t \geq 0}\|v\|_{\dot{B}_{p, \infty}^{-2+\frac{n}{p}}}+$ $\sup _{t>0} t^{1-\frac{n}{2 p}}\|v\|_{L^{p}}$, respectively, $\mathcal{X}_{p}$ and $\mathcal{Y}_{p}$ are Banach spaces.

The main results of this paper are as follows:

Theorem 1.1. Let $\left(v_{0}, w_{0}\right) \in\left(L^{\frac{n}{2}}\left(\mathbb{R}^{n}\right)\right)^{2}$ be such that $\left\|\left(v_{0}, w_{0}\right)\right\|_{L^{\frac{n}{2}}} \leq \varepsilon$, where $\varepsilon$ is as above. Let $p \in\left(\frac{n}{2}, n\right)$ and $(v, w) \in\left(\mathcal{X}_{p}\right)^{2}$ be the solution of $(1.1)-(1.4)$ mentioned above. There exist constants $K_{1}$ and $K_{2}$ depending only on $n, p$ and $\varepsilon$ such that

$$
\left\|\left(\partial_{x}^{\beta} v(t), \partial_{x}^{\beta} w(t)\right)\right\|_{L^{q}} \leq K_{1}\left(K_{2}|\beta|\right)^{|\beta|} t^{-\frac{|\beta|}{2}-1+\frac{n}{2 q}}
$$

for all $\frac{n}{2} \leq q \leq \infty, t>0$ and $\beta \in \mathbb{N}_{0}^{n}$.

Theorem 1.2. Let $p \in\left(\frac{n}{2}, n\right)$, and let $\left(v_{0}, w_{0}\right) \in\left(\dot{B}_{p, \infty}^{-2+\frac{n}{p}}\left(\mathbb{R}^{n}\right)\right)^{2}$ be such that $\left\|\left(v_{0}, w_{0}\right)\right\|_{\dot{B}_{p, \infty}^{-2+\frac{n}{p}}} \leq \varepsilon$, where $\varepsilon$ is as above. Let $(v, w) \in\left(\mathcal{Y}_{p}\right)^{2}$ be the solution of (1.1)-(1.4) mentioned above. There exist constants $K_{1}$ and $K_{2}$ depending only on $n, p$ and $\varepsilon$ such that

$$
\left\|\left(\partial_{x}^{\beta} v(t), \partial_{x}^{\beta} w(t)\right)\right\|_{L^{q}} \leq K_{1}\left(K_{2}|\beta|\right)^{|\beta|} t^{-\frac{|\beta|}{2}-1+\frac{n}{2 q}}
$$

for all $p \leq q \leq \infty, t>0$ and $\beta \in \mathbb{N}_{0}^{n}$.

Remark 1.3. (i) The inequality (1.8) shows that mild solutions of (1.1)-(1.4) with initial data in $\left(L^{\frac{n}{2}}\left(\mathbb{R}^{n}\right)\right)^{2}$ are spatially analytic, and the $L^{q}$-norm $\left(\frac{n}{2} \leq\right.$ $q \leq \infty)$ of its $\beta$ th order spatial derivative decay to zero as $t \rightarrow \infty$ in a rate of the form $t^{-\frac{|\beta|}{2}-1+\frac{n}{2 q}}$. The inequality (1.9) shows that similar results hold for mild solutions of $(1.1)-(1.4)$ with initial data in $\left(\dot{B}_{p, \infty}^{-2+\frac{n}{p}}\left(\mathbb{R}^{n}\right)\right)^{2}$. 
(ii) As was shown in [22], for large initial data $\left(v_{0}, w_{0}\right) \in\left(L^{\frac{n}{2}}\left(\mathbb{R}^{n}\right)\right)^{2}$, there exists $T>0$ such that the system (1.1)-(1.4) has a unique local-in-time mild solution. For a such solution, we have a similar estimate as (1.8), but with $t>0$ replaced by $t \in(0, T)$ and the constants $K_{1}$ and $K_{2}$ depending also on $T$. A similar remark can be made to Theorem 1.2 for solutions whose initial data belong to the closure of $\left(\mathcal{S}\left(\mathbb{R}^{n}\right)\right)^{2}$ in $\left(\dot{B}_{p, \infty}^{-2+\frac{n}{p}}\left(\mathbb{R}^{n}\right)\right)^{2}$, where $\mathcal{S}\left(\mathbb{R}^{n}\right)$ is the Schwartz space.

The remaining part of this paper is organized as follows. In Section 2, we state some preliminary results. Section 3 is devoted to presenting the proof of Theorem 1.1. Section 4 is devoted to presenting the proof of Theorem 1.2.

\section{Preliminaries}

In this section we collect some preliminary materials for the reader's convenience. We first recall the definition of the homogeneous Besov space $\dot{B}_{p, \infty}^{-\alpha}\left(\mathbb{R}^{n}\right)$ in the version of the heat kernel characterization of this space:

Definition 2.1. (cf. [14]) Let $\alpha>0$ and $1 \leq p \leq \infty$. We define $\dot{B}_{p, \infty}^{-\alpha}\left(\mathbb{R}^{n}\right)=\left\{u \in \mathcal{S}^{\prime}\left(\mathbb{R}^{n}\right): e^{t \Delta} u \in C\left((0, \infty), L^{p}\left(\mathbb{R}^{n}\right)\right)\right.$ and $\left.\sup _{t>0} t^{\frac{\alpha}{2}}\left\|e^{t \Delta} u\right\|_{L^{p}}<\infty\right\}$ with norm

$$
\|u\|_{\dot{B}_{p, \infty}^{-\alpha}}=\sup _{t>0} t^{\frac{\alpha}{2}}\left\|e^{t \Delta} u\right\|_{L^{p}}
$$

Here $\mathcal{S}^{\prime}\left(\mathbb{R}^{n}\right)$ represents the dual of the Schwartz space, i.e., the space of tempered distributions. It is well-known that $\left(\dot{B}_{p, \infty}^{-\alpha}\left(\mathbb{R}^{n}\right),\|\cdot\|_{\dot{B}_{p, \infty}^{-\alpha}}\right)$ is a Banach space (cf. [14]).

Next we give the regularizing and decay rate estimates for the heat operator $e^{t \Delta}$ :

Lemma 2.2. (i) Let $1 \leq p \leq q \leq \infty$. Then for any $u \in L^{p}\left(\mathbb{R}^{n}\right)$ we have

$$
\left\|\partial_{x}^{\beta} e^{t \Delta} u\right\|_{L^{q}} \leq C(n)|\beta|^{\frac{|\beta|}{2}} t^{-\frac{|\beta|}{2}-\frac{n}{2}\left(\frac{1}{p}-\frac{1}{q}\right)}\|u\|_{L^{p}} \quad \text { for all } t>0, \beta \in \mathbb{N}_{0}^{n}
$$

(ii) Let $\frac{n}{2}<p \leq q \leq \infty$. Then for any $u \in \dot{B}_{p, \infty}^{-2+\frac{n}{p}}\left(\mathbb{R}^{n}\right)$ we have

$$
\left\|\partial_{x}^{\beta} e^{t \Delta} u\right\|_{L^{q}} \leq C(n)|\beta|^{\frac{|\beta|}{2}} t^{-\frac{|\beta|}{2}-1+\frac{n}{2 q}}\|u\|_{\dot{B}_{p, \infty}^{-2+\frac{n}{p}}} \quad \text { for all } t>0, \beta \in \mathbb{N}_{0}^{n} \text {. }
$$

Here $C(n)$ is a constant depending only on $n$.

Proof. For (i), we refer the reader to see Lemma 2.1 of [8]. To prove (ii) we observe that since $e^{t \Delta}$ is the convolution operator with kernel $G(x, t)=$ $(4 \pi t)^{-\frac{n}{2}} \exp \left(-\frac{|x|^{2}}{4 t}\right)$, the Young inequality yields

$$
\left\|\partial_{x} e^{t \Delta} u\right\|_{L^{q}} \leq\left\|\partial_{x} G(x, t)\right\|_{L^{1}}\|u\|_{L^{q}} \leq \pi^{-\frac{1}{2}} t^{-\frac{1}{2}}\|u\|_{L^{q}}
$$


On the other hand, it can be easily seen that

$$
\partial_{x}^{\beta} e^{t \Delta} u=\prod_{i=1}^{n}\left(\partial_{x_{i}} e^{\frac{t}{2|\beta|} \Delta}\right)^{\beta_{i}} e^{\frac{t}{2} \Delta} u
$$

Hence, using (2.3), (2.4) and Definition 2.1 we get

$$
\begin{aligned}
\left\|\partial_{x}^{\beta} e^{t \Delta} u\right\|_{L^{q}} & \leq \prod_{i=1}^{n}\left\|\partial_{x_{i}} e^{\frac{t}{2|\beta|} \Delta}\right\|_{\mathcal{L}_{i}\left(L^{q}, L^{q}\right)}\left\|e^{\frac{t}{2} \Delta} u\right\|_{L^{q}} \\
& \leq C(n)\left[\pi^{-\frac{1}{2}}\left(\frac{t}{2|\beta|}\right)^{-\frac{1}{2}}\right]^{|\beta|}\left(\frac{t}{4}\right)^{-\frac{n}{2}\left(\frac{1}{p}-\frac{1}{q}\right)}\left\|e^{\frac{t}{4} \Delta} u\right\|_{L^{p}} \\
& \leq C(n)\left[\pi^{-\frac{1}{2}}\left(\frac{t}{2|\beta|}\right)^{-\frac{1}{2}}\right]^{|\beta|}\left(\frac{t}{4}\right)^{-\frac{n}{2}\left(\frac{1}{p}-\frac{1}{q}\right)}\left(\frac{t}{4}\right)^{-1+\frac{n}{2 p}}\left(\frac{t}{4}\right)^{1-\frac{n}{2 p}}\left\|e^{\frac{t}{4} \Delta} u\right\|_{L^{p}} \\
& \leq C(n)|\beta|^{\frac{|\beta|}{2}} t^{-\frac{|\beta|}{2}-1+\frac{n}{2 q}} \sup _{\frac{t}{4}>0}\left(\frac{t}{4}\right)^{1-\frac{n}{2 p}}\left\|e^{\frac{t}{4} \Delta} u\right\|_{L^{p}} \\
& \leq C(n)|\beta|^{\mid \frac{|\beta|}{2}} t^{-\frac{|\beta|}{2}-1+\frac{n}{2 q}}\|u\|_{\dot{B}_{p, \infty}^{-2+\frac{n}{p}}}
\end{aligned}
$$

Here $\|\mathbf{T}\|_{\mathcal{L}\left(L^{p}, L^{q}\right)}$ denotes the operator norm of the linear operator $\mathbf{T}$ : $L^{p}\left(\mathbb{R}^{n}\right) \rightarrow L^{q}\left(\mathbb{R}^{n}\right)$. This finishes the proof of Lemma 2.2 (ii). (cf. [14]):

The following is the well-known Hardy-Littlewood-Sobolev inequality

Lemma 2.3. For any $1<p<n$, the operator $(-\Delta)^{-\frac{1}{2}}$ is bounded from $L^{p}\left(\mathbb{R}^{n}\right)$ to $L^{\frac{n p}{n-p}}\left(\mathbb{R}^{n}\right)$, i.e., for any $u \in L^{p}\left(\mathbb{R}^{n}\right)$ we have

$$
\left\|(-\Delta)^{-\frac{1}{2}} u\right\|_{L^{\frac{n p}{n-p}}} \leq C(n, p)\|u\|_{L^{p}},
$$

where $C(n, p)$ is a constant depending only on $n$ and $p$.

The following preliminary result is due to C. Kahane (cf. Lemma 2.1 of $[10])$ :

Lemma 2.4. Let $\delta>\frac{1}{2}$. Then there exists a positive constant $C$ depending only on $\delta$ such that

$$
\sum_{\alpha \leq \beta}\left(\begin{array}{l}
\beta \\
\alpha
\end{array}\right)|\alpha|^{|\alpha|-\delta}|\beta-\alpha|^{|\beta-\alpha|-\delta} \leq C(\delta)|\beta|^{|\beta|-\delta} \quad \text { for all } \beta \in \mathbb{N}_{0}^{n} .
$$

Here, the notation $\alpha \leq \beta$ means that $\alpha_{i} \leq \beta_{i}$ for all $i=1,2, \ldots, n$, and $\left(\begin{array}{l}\beta \\ \alpha\end{array}\right)=\prod_{i=1}^{n} \frac{\beta_{i} !}{\alpha_{i} !\left(\beta_{i}-\alpha_{i}\right) !}$. Note that the dependence of $C(\delta)$ on $\delta$ is merely of the form $\sum_{j=1}^{\infty} j^{-\delta-\frac{1}{2}}$.

Finally we recall the following useful lemma, whose proof can be found in $[8]$ :

Lemma 2.5. Let $\psi_{0}$ be a measurable and locally bounded function in $(0, \infty)$. Let $\left\{\psi_{j}\right\}_{j=1}^{\infty}$ be a sequence of measurable functions in $(0, \infty)$. Assume that $\alpha \in \mathbb{R}$ and $\mu, \nu>0$ satisfying $\mu+\nu=1$. Let $B_{\eta}>0$ be a number depending on 
$\eta \in(0,1)$, and assume that $B_{\eta}$ is nonincreasing with respect to $\eta$. Assume that there is a positive constant $\sigma$ such that

$$
0 \leq \psi_{0}(t) \leq B_{\eta} t^{-\alpha}+\sigma \int_{(1-\eta) t}^{t}(t-\tau)^{-\mu} \tau^{-\nu} \psi_{0}(\tau) d \tau
$$

and

$$
0 \leq \psi_{j+1}(t) \leq B_{\eta} t^{-\alpha}+\sigma \int_{(1-\eta) t}^{t}(t-\tau)^{-\mu} \tau^{-\nu} \psi_{j}(\tau) d \tau
$$

for all $j \geq 0, t>0$ and $\eta \in(0,1)$. Let $\eta_{0}$ be a unique positive number such that $I\left(\eta_{0}\right)=\min \left\{\frac{1}{2 \sigma}, I(1)\right\}$ with $I(\eta)=\int_{1-\eta}^{1}(1-\tau)^{-\mu} \tau^{-\alpha-\nu} d \tau$. Then

$$
\psi_{j}(t) \leq 2 B_{\eta_{0}} t^{-\alpha} \quad \text { for all } j \geq 0 \text { and } t>0 .
$$

Remark 2.6. From the proof of the above lemma (see [8]) we see that in (2.9), $\eta_{0}$ can be replaced with any $0<\eta \leq \eta_{0}$.

\section{Proof of Theorem 1.1}

In this section we give the proof of Theorem 1.1. We follow the idea of [8] and first prove a variant of Theorem 1.1 under some additional regularity assumptions. In what follows, we shall use notations $C_{i}(a, b, \ldots)$ and $\tilde{C}_{i}(a, b, \ldots)$ to denote various constants which depend only on the indicated parameters $a, b, \ldots$

Proposition 3.1. Let the assumptions of Theorem 1.1 be satisfied. Furthermore, we assume that

$$
\left(\partial_{x}^{\beta} v(t), \partial_{x}^{\beta} w(t)\right) \in\left(C\left((0, \infty), L^{q}\left(\mathbb{R}^{n}\right)\right)\right)^{2}
$$

for all $\frac{n}{2} \leq q \leq \infty$ and $\beta \in \mathbb{N}_{0}^{n}$. Then for any $\delta \in\left(\frac{1}{2}, 1\right]$, there exist positive constants $K_{1}$ and $K_{2}$ depending only on $n, p, \varepsilon$ and $\delta$ such that

$$
\left\|\left(\partial_{x}^{\beta} v(t), \partial_{x}^{\beta} w(t)\right)\right\|_{L^{q}} \leq K_{1}\left(K_{2}|\beta|\right)^{|\beta|-\delta} t^{-\frac{|\beta|}{2}-1+\frac{n}{2 q}}
$$

for all $\frac{n}{2} \leq q \leq \infty, t>0$ and $\beta \in \mathbb{N}_{0}^{n}$.

Proof. We deduce by induction on $m=|\beta|$ and split the proof into two steps.

Step 1. We first prove (3.2) for $m=0$. Note that from [22] we know that for any $\left(v_{0}, w_{0}\right) \in\left(L^{\frac{n}{2}}\left(\mathbb{R}^{n}\right)\right)^{2}$ with $\left\|\left(v_{0}, w_{0}\right)\right\|_{L^{\frac{n}{2}}} \leq \varepsilon$, the corresponding mild solution satisfies $\|(v(t), w(t))\|_{\mathcal{X}_{p}} \leq C \varepsilon$ for some universal constant $C$, i.e.,

$$
\sup _{t \geq 0}\|(v(t), w(t))\|_{L^{\frac{n}{2}}}+\sup _{t>0} t^{1-\frac{n}{2 p}}\|(v(t), w(t))\|_{L^{p}} \leq C \varepsilon .
$$

Hence (3.2) is trivial if either $q=\frac{n}{2}$ or $q=p$. Moreover, by interpolation, (3.2) also immediately follows if $q \in\left(\frac{n}{2}, p\right)$. In what follows we consider the case $q \in(p, \infty]$. Let $\eta \in(0,1)$ be a constant to be specified later. By taking 
$L^{q}$-norm of both sides of (1.6) and dividing the time integral into two parts, we have

$$
\begin{aligned}
\|v(t)\|_{L^{q}} \leq & \left\|e^{t \Delta} v_{0}\right\|_{L^{q}}+\left(\int_{0}^{t(1-\eta)}+\int_{t(1-\eta)}^{t}\right) \| e^{(t-\tau) \Delta} \nabla \\
& \cdot\left[v \nabla\left((-\Delta)^{-1}(w-v)\right)\right] \|_{L^{q}}(\tau) d \tau \\
= & A_{1}+A_{2}+A_{3} .
\end{aligned}
$$

We shall estimate the three terms one by one. For $A_{1}$, by Lemma 2.2 (i), we easily see that

$$
A_{1} \leq C_{1}(n) t^{-1+\frac{n}{2 q}}\left\|v_{0}\right\|_{L^{\frac{n}{2}}} \leq C_{1}(n, \varepsilon) t^{-1+\frac{n}{2 q}} .
$$

For $A_{2}$, by Lemma 2.2 (i), Lemma 2.3 and (3.3) we have

$$
\begin{aligned}
A_{2}= & \int_{0}^{t(1-\eta)}\left\|e^{(t-\tau) \Delta} \nabla \cdot\left[v \nabla\left((-\Delta)^{-1}(w-v)\right)\right]\right\|_{L^{q}}(\tau) d \tau \\
\leq & C_{2}(n) \int_{0}^{t(1-\eta)}(t-\tau)^{-\frac{1}{2}-\frac{n}{2}\left(\frac{2}{n}+\frac{1}{p}-\frac{1}{n}-\frac{1}{q}\right)}\|v(\tau)\|_{L^{\frac{n}{2}}} \\
& \times\left\|\nabla\left((-\Delta)^{-1}(w-v)\right)(\tau)\right\|_{L^{\frac{n p}{n-p}}} d \tau \\
\leq & C_{2}(n, p) \int_{0}^{t(1-\eta)}(t-\tau)^{-1-\frac{n}{2 p}+\frac{n}{2 q}}\|v(\tau)\|_{L^{\frac{n}{2}}}\|(v(\tau), w(\tau))\|_{L^{p}} d \tau \\
\leq & C_{2}(n, p) \varepsilon^{2} \int_{0}^{t(1-\eta)}(t-\tau)^{-1-\frac{n}{2 p}+\frac{n}{2 q}} \tau^{-1+\frac{n}{2 p}} d \tau \\
\leq & C_{2}(n, p, \varepsilon) \eta^{-2} t^{-1+\frac{n}{2 q}} .
\end{aligned}
$$

For $A_{3}$, by using Lemmas 2.2 (i), 2.3 and (3.3) again we see that

$$
\begin{aligned}
A_{3}= & \int_{t(1-\eta)}^{t}\left\|e^{(t-\tau) \Delta} \nabla \cdot\left[v \nabla\left((-\Delta)^{-1}(w-v)\right)\right]\right\|_{L^{q}}(\tau) d \tau \\
\leq & C_{3}(n) \int_{t(1-\eta)}^{t}(t-\tau)^{-\frac{1}{2}-\frac{n}{2}\left(\frac{1}{q}+\frac{1}{p}-\frac{1}{n}-\frac{1}{q}\right)}\|v(\tau)\|_{L^{q}} \\
& \times\left\|\nabla\left((-\Delta)^{-1}(w-v)\right)(\tau)\right\|_{L^{\frac{n p}{n-p}}} d \tau \\
\leq & C_{3}(n, p) \int_{t(1-\eta)}^{t}(t-\tau)^{-\frac{n}{2 p}}\|v(\tau)\|_{L^{q}}\|(v(\tau), w(\tau))\|_{L^{p}} d \tau \\
\leq & C_{3}(n, p, \varepsilon) \int_{t(1-\eta)}^{t}(t-\tau)^{-\frac{n}{2 p}} \tau^{-1+\frac{n}{2 p}}\|v(\tau)\|_{L^{q}} d \tau .
\end{aligned}
$$

Combining (3.5)-(3.7) and denoting $\bar{B}_{\eta}=C_{1}(n, \varepsilon)+C_{2}(n, p, \varepsilon) \eta^{-2}$, we see that (3.4) yields

$$
\|v(t)\|_{L^{q}} \leq \bar{B}_{\eta} t^{-1+\frac{n}{2 q}}+C_{3}(n, p, \varepsilon) \int_{t(1-\eta)}^{t}(t-\tau)^{-\frac{n}{2 p}} \tau^{-1+\frac{n}{2 p}}\|v(\tau)\|_{L^{q}} d \tau .
$$


Similarly we can deal with (1.7) to obtain that

$$
\|w(t)\|_{L^{q}} \leq \bar{B}_{\eta} t^{-1+\frac{n}{2 q}}+C_{3}(n, p, \varepsilon) \int_{t(1-\eta)}^{t}(t-\tau)^{-\frac{n}{2 p}} \tau^{-1+\frac{n}{2 p}}\|w(\tau)\|_{L^{q}} d \tau
$$

From (3.8) and (3.9) we have

$\|(v(t), w(t))\|_{L^{q}} \leq B_{\eta} t^{-1+\frac{n}{2 q}}+C_{4} \int_{t(1-\eta)}^{t}(t-\tau)^{-\frac{n}{2 p}} \tau^{-1+\frac{n}{2 p}}\|(v(\tau), w(\tau))\|_{L^{q}} d \tau$

where $B_{\eta}=2 \bar{B}_{\eta}$ and $C_{4}=2 C_{3}(n, p, \varepsilon)$. By applying Lemma 2.5, we get the desired estimate $(3.2)$ for $|\beta|=m=0$ with $K_{1}=2 B_{\eta_{0}}$, where $\eta_{0}=$ $\eta_{0}(n, p, \varepsilon) \in(0,1)$.

Step 2. Next we prove (3.2) for $m \geq 1$. Since the arguments for the cases $\frac{n}{2} \leq q<n$ and $n \leq q \leq \infty$ are different, we consider the two different cases separately.

Case 1: $\frac{n}{2} \leq q<n$. In this case, we first differentiate (1.6) to get

$$
\partial_{x}^{\beta} v(t)=\partial_{x}^{\beta} e^{t \Delta} v_{0}-\int_{0}^{t} \partial_{x}^{\beta} e^{(t-\tau) \Delta} \nabla \cdot\left[v \nabla\left((-\Delta)^{-1}(w-v)\right)\right](\tau) d \tau .
$$

For $\eta \in(0,1)$ to be chosen later, we split the time integral into two parts and get:

$$
\begin{aligned}
\left\|\partial_{x}^{\beta} v(t)\right\|_{L^{q}} \leq & \left\|\partial_{x}^{\beta} e^{t \Delta} v_{0}\right\|_{L^{q}}+\left(\int_{0}^{t(1-\eta)}+\int_{t(1-\eta)}^{t}\right) \\
& \times\left\|\partial_{x}^{\beta} e^{(t-\tau) \Delta} \nabla \cdot\left[v \nabla\left((-\Delta)^{-1}(w-v)\right)\right]\right\|_{L^{q}}(\tau) d \tau \\
= & B_{1}+B_{2}+B_{3} .
\end{aligned}
$$

For $B_{1}$, by Lemma 2.2 (i) we have

$$
B_{1} \leq C_{5}(n) m^{\frac{m}{2}} t^{-\frac{m}{2}-1+\frac{n}{2 q}}\left\|v_{0}\right\|_{L^{\frac{n}{2}}} \leq C_{5}(n, \varepsilon) m^{m-\delta} t^{-\frac{m}{2}-1+\frac{n}{2 q}}
$$

Here we used the evident facts that $m^{\frac{m}{2}}=m^{m-\delta}$ for $m=1$, and $\frac{m}{2} \leq$ $m-\delta$ for $m \geq 2$ and $\delta \in\left(\frac{1}{2}, 1\right]$. For $B_{2}$, using Lemmas 2.2 (i), 2.3 and (3.3), 
we have

$$
\begin{aligned}
B_{2}= & \int_{0}^{t(1-\eta)}\left\|\partial_{x}^{\beta} e^{(t-\tau) \Delta} \nabla \cdot\left[v \nabla\left((-\Delta)^{-1}(w-v)\right)\right]\right\|_{L^{q}}(\tau) d \tau \\
\leq & C_{6}(n, p) \int_{0}^{t(1-\eta)}\left\|e^{\frac{t-\tau}{2} \Delta} \nabla\right\|_{\mathcal{L}\left(L^{q}, L^{q}\right)} \\
& \left\|\partial_{x}^{\beta} e^{\frac{t-\tau}{2} \Delta}\right\|_{\mathcal{L}\left(L^{\frac{n p}{n+p}}, L^{q}\right)}\|v(\tau)\|_{L^{\frac{n}{2}}}\|(v(\tau), w(\tau))\|_{L^{p}} d \tau \\
\leq & C_{6}(n, p) m^{\frac{m}{2}} \int_{0}^{t(1-\eta)}\left(\frac{t-\tau}{2}\right)^{-\frac{m}{2}-1-\frac{n}{2 p}+\frac{n}{2 q}}\|v(\tau)\|_{L^{\frac{n}{2}}}\|(v(\tau), w(\tau))\|_{L^{p}} d \tau \\
\leq & C_{6}(n, p) \varepsilon^{2} m^{\frac{m}{2}} \int_{0}^{t(1-\eta)}\left(\frac{t-\tau}{2}\right)^{-\frac{m}{2}-1-\frac{n}{2 p}+\frac{n}{2 q}} \tau^{-1+\frac{n}{2 p}} d \tau \\
\leq & C_{6}(n, p, \varepsilon)(2 m)^{\frac{m}{2}} \eta^{-\frac{m}{2}-\frac{3}{2}} t^{-\frac{m}{2}-1+\frac{n}{2 q}} .
\end{aligned}
$$

For $B_{3}$, by using the Leibniz rule we have

$$
\begin{aligned}
B_{3}= & \int_{t(1-\eta)}^{t}\left\|\partial_{x}^{\beta} e^{(t-\tau) \Delta} \nabla \cdot\left[v \nabla\left((-\Delta)^{-1}(w-v)\right)\right]\right\|_{L^{q}}(\tau) d \tau \\
\leq & \int_{t(1-\eta)}^{t}\left\|e^{\frac{t-\tau}{2} \Delta} \nabla\right\|_{\mathcal{L}\left(L^{q}, L^{q}\right)}\left\|e^{\frac{t-\tau}{2} \Delta} \partial_{x}^{\beta}\left[v \nabla\left((-\Delta)^{-1}(w-v)\right)\right]\right\|_{L^{q}}(\tau) d \tau \\
\leq & C_{7}(n) \int_{t(1-\eta)}^{t}\left(\frac{t-\tau}{2}\right)^{-\frac{1}{2}}\left\|e^{\frac{t-\tau}{2} \Delta} \partial_{x}^{\beta}\left[v \nabla\left((-\Delta)^{-1}(w-v)\right)\right]\right\|_{L^{q}}(\tau) d \tau \\
\leq & C_{7}(n) \int_{t(1-\eta)}^{t}\left(\frac{t-\tau}{2}\right)^{-\frac{1}{2}}\left\|e^{\frac{t-\tau}{2} \Delta}\left[\left(\partial_{x}^{\beta} v\right) \nabla\left((-\Delta)^{-1}(w-v)\right)\right]\right\|_{L^{q}}(\tau) d \tau \\
& +C_{7}(n) \int_{t(1-\eta)}^{t}\left(\frac{t-\tau}{2}\right)^{-\frac{1}{2}}\left\|e^{\frac{t-\tau}{2} \Delta}\left[\sum_{0<\gamma<\beta}\left(\begin{array}{c}
\beta \\
\gamma
\end{array}\right)\left(\partial_{x}^{\gamma} v\right)\left(\partial_{x}^{\beta-\gamma} \nabla\left((-\Delta)^{-1}(w-v)\right)\right)\right]\right\|_{L^{q}}(\tau) d \tau \\
& +C_{7}(n) \int_{t(1-\eta)}^{t}\left(\frac{t-\tau}{2}\right)^{-\frac{1}{2}}\left\|e^{\frac{t-\tau}{2} \Delta}\left[v \partial_{x}^{\beta}\left(\nabla\left((-\Delta)^{-1}(w-v)\right)\right)\right]\right\|_{L^{q}}(\tau) d \tau \\
= & B_{31}+B_{32}+B_{33} .
\end{aligned}
$$

Here, the notation $\gamma<\beta$ means that $\gamma \leq \beta$ and $|\gamma|<|\beta|$. For $B_{31}$, Lemma 2.2 (i) implies that

$$
\begin{aligned}
B_{31} & \leq C_{8}(n, p) \int_{t(1-\eta)}^{t}\left(\frac{t-\tau}{2}\right)^{-\frac{1}{2}-\frac{n}{2}\left(\frac{1}{q}+\frac{1}{p}-\frac{1}{n}-\frac{1}{q}\right)}\left\|\partial_{x}^{\beta} v(\tau)\right\|_{L^{q}}\|(v(\tau), w(\tau))\|_{L^{p}} d \tau \\
& \leq C_{8}(n, p, \varepsilon) \int_{t(1-\eta)}^{t}(t-\tau)^{-\frac{n}{2 p}} \tau^{-1+\frac{n}{2 p}}\left\|\partial_{x}^{\beta} v(\tau)\right\|_{L^{q}} d \tau
\end{aligned}
$$


For $B_{32}$, by the induction assumption and Lemma 2.4 we get

$$
\begin{aligned}
B_{32} \leq & C_{9}(n, p) \int_{t(1-\eta)}^{t}\left(\frac{t-\tau}{2}\right)^{-\frac{n}{2 p}} \sum_{0<\gamma<\beta}\left(\begin{array}{l}
\beta \\
\gamma
\end{array}\right)\left\|\partial_{x}^{\gamma} v(\tau)\right\|_{L^{q}} \\
& \times\left\|\left(\partial_{x}^{\beta-\gamma} v(\tau), \partial_{x}^{\beta-\gamma} w(\tau)\right)\right\|_{L^{p}} d \tau \\
\leq & C_{9}(n, p) \int_{t(1-\eta)}^{t}\left(\frac{t-\tau}{2}\right)^{-\frac{n}{2 p}}\left(\sum_{0<\gamma<\beta}\left(\begin{array}{l}
\beta \\
\gamma
\end{array}\right) K_{1}\left(K_{2}|\gamma|\right)^{|\gamma|-\delta} \tau^{-\frac{|\gamma|}{2}-1+\frac{n}{2 q}}\right. \\
& \left.\times K_{1}\left(K_{2}|\beta-\gamma|\right)^{|\beta-\gamma|-\delta} \tau^{-\frac{|\beta-\gamma|}{2}-1+\frac{n}{2 p}}\right) d \tau \\
\leq & C_{9}(n, p) K_{1}^{2} K_{2}^{m-2 \delta}\left(\sum_{0<\gamma<\beta}\left(\begin{array}{l}
\beta \\
\gamma
\end{array}\right)|\gamma|^{|\gamma|-\delta}|\beta-\gamma|^{|\beta-\gamma|-\delta}\right) \\
& \times \int_{t(1-\eta)}^{t}(t-\tau)^{-\frac{n}{2 p}} \tau^{-\frac{m}{2}-2+\frac{n}{2 q}+\frac{n}{2 p}} d \tau \\
\leq & C_{9}(n, p, \delta) K_{1}^{2} K_{2}^{m-2 \delta} m^{m-\delta} I(\eta) t^{-\frac{m}{2}-1+\frac{n}{2 q}}
\end{aligned}
$$

where $I(\eta)=\int_{1-\eta}^{1}(1-\tau)^{-\frac{n}{2 p}} \tau^{-\frac{m}{2}-2+\frac{n}{2 q}+\frac{n}{2 p}} d \tau$. For $B_{33}$, using Lemma 2.2 (i) again, the Calderón-Zygmund theorem, the result of Step 1 and the induction assumption, we obtain

$$
\begin{aligned}
B_{33} & \leq C_{10}(n, p) \int_{t(1-\eta)}^{t}\left(\frac{t-\tau}{2}\right)^{-\frac{n}{2 p}}\|v(\tau)\|_{L^{\frac{n q}{n-q}}}\left\|\partial_{x}^{\beta}\left(\nabla\left((-\Delta)^{-1}(w-v)\right)\right)\right\|_{L^{p}} d \tau \\
& \leq C_{10}(n, p) \int_{t(1-\eta)}^{t}\left(\frac{t-\tau}{2}\right)^{-\frac{n}{2 p}}\|v(\tau)\|_{L^{\frac{n q}{n-q}}}\left\|\left(\partial_{x}^{\beta-1} v(\tau), \partial_{x}^{\beta-1} w(\tau)\right)\right\|_{L^{p}} d \tau \\
& \leq C_{10}(n, p, \delta) K_{1}^{2} K_{2}^{m-2 \delta} m^{m-\delta} I(\eta) t^{-\frac{m}{2}-1+\frac{n}{2 q}}
\end{aligned}
$$

Combining estimates (3.12)-(3.17) and denoting

$$
\begin{aligned}
\bar{B}_{\eta}= & C_{5}(n, \varepsilon) m^{m-\delta}+C_{6}(n, p, \varepsilon)(2 m)^{\frac{m}{2}} \eta^{-\frac{m}{2}-\frac{3}{2}} \\
& +C_{11}(n, p, \delta) K_{1}^{2} K_{2}^{m-2 \delta} m^{m-\delta} I(\eta),
\end{aligned}
$$

where $C_{11}(n, p, \delta)=C_{9}(n, p, \delta)+C_{10}(n, p, \delta)$, we see that

$$
\begin{aligned}
\left\|\partial_{x}^{\beta} v(t)\right\|_{L^{q}} \leq & \bar{B}_{\eta} t^{-\frac{m}{2}-1+\frac{n}{2 q}} \\
& +C_{8}(n, p, \varepsilon) \int_{t(1-\eta)}^{t}(t-\tau)^{-\frac{n}{2 p}} \tau^{-1+\frac{n}{2 p}}\left\|\left(\partial_{x}^{\beta} v(\tau), \partial_{x}^{\beta} w(\tau)\right)\right\|_{L^{q}} d \tau .
\end{aligned}
$$


The estimate for $\partial_{x}^{\beta} w(t)$ is similar, so that

$$
\begin{aligned}
\left\|\partial_{x}^{\beta} w(t)\right\|_{L^{q}} \leq & \bar{B}_{\eta} t^{-\frac{m}{2}-1+\frac{n}{2 q}} \\
& +C_{8}(n, p, \varepsilon) \int_{t(1-\eta)}^{t}(t-\tau)^{-\frac{n}{2 p}} \tau^{-1+\frac{n}{2 p}}\left\|\left(\partial_{x}^{\beta} v(\tau), \partial_{x}^{\beta} w(\tau)\right)\right\|_{L^{q}} d \tau .
\end{aligned}
$$

From (3.18) and (3.19) we obtain

$$
\begin{aligned}
\left\|\left(\partial_{x}^{\beta} v(t), \partial_{x}^{\beta} w(t)\right)\right\|_{L^{q}} \leq & B_{\eta} t^{-\frac{m}{2}-1+\frac{n}{2 q}} \\
& +C_{12} \int_{t(1-\eta)}^{t}(t-\tau)^{-\frac{n}{2 p}} \tau^{-1+\frac{n}{2 p}}\left\|\left(\partial_{x}^{\beta} v(\tau), \partial_{x}^{\beta} w(\tau)\right)\right\|_{L^{q}} d \tau .
\end{aligned}
$$

Here $B_{\eta}=2 \bar{B}_{\eta}$ and $C_{12}=2 C_{8}(n, p, \varepsilon)$. Hence, by Lemma 2.5 and Remark 2.6, there exists $\eta_{m_{0}}$ such that for any $0<\eta_{m} \leq \eta_{m_{0}}$,

$$
\left\|\left(\partial_{x}^{\beta} v(t), \partial_{x}^{\beta} w(t)\right)\right\|_{L^{q}} \leq 2 B_{\eta_{m}} t^{-\frac{m}{2}-1+\frac{n}{2 q}} \quad \text { for all } t>0 .
$$

Let $\eta_{m}=\frac{1}{m}$. Since $I\left(\eta_{m}\right)$ is strictly monotone decreasing in $m$ and $I\left(\eta_{m}\right) \rightarrow 0$ as $m \rightarrow \infty$, we can choose $m_{0}$ sufficiently large such that $I\left(\frac{1}{m}\right) \leq \frac{1}{2 C_{12}}$ for all $m \geq m_{0}$. Hence, we obtain

$$
\left\|\left(\partial_{x}^{\beta} v(t), \partial_{x}^{\beta} w(t)\right)\right\|_{L^{q}} \leq 2 B_{\frac{1}{m}} t^{-\frac{m}{2}-1+\frac{n}{2 q}} \quad \text { for all } t>0 \text { and }|\beta|=m .
$$

By (3.21), we can choose $K_{1}$ and $K_{2}$ sufficiently large such that (3.2) holds for all $\beta$ satisfying $|\beta| \leq m_{0}$. Hence, to get the desired assertion it suffices to prove that it is possible to choose $K_{1}$ and $K_{2}$ sufficiently large such that also $2 B_{\frac{1}{m}} \leq K_{1}\left(K_{2} m\right)^{m-\delta}$ for all $m>m_{0}$. For this purpose we note that it is clear that

$$
I\left(\frac{1}{m}\right)=\int_{1-\frac{1}{m}}^{1}(1-\tau)^{-\frac{n}{2 p}} \tau^{-\frac{m}{2}-2+\frac{n}{2 q}+\frac{n}{2 p}} d \tau \leq 2 \sqrt{e} \leq 4 \quad \text { for all } m \geq 1,
$$

so that we can calculate $2 B_{\frac{1}{m}}$ as follows:

$$
2 B_{\frac{1}{m}} \leq 4\left[C_{5}+C_{6} 2^{\frac{m}{2}} m^{\delta+\frac{3}{2}}+4 C_{11} K_{1}^{2} K_{2}^{m-2 \delta}\right] m^{m-\delta} .
$$

Note that there exists a constant $C_{13}>2$ such that $2^{\frac{m}{2}} m^{\delta+\frac{3}{2}} \leq 2^{m-\delta} m^{\delta+\frac{3}{2}} \leq$ $C_{13}^{m-\delta}$. Hence,

$$
\begin{aligned}
2 B_{\frac{1}{m}} & \leq 4\left[C_{5}+C_{6} C_{13}^{m-\delta}+4 C_{11} K_{1}^{2} K_{2}^{m-2 \delta}\right] m^{m-\delta} \\
& \leq 4\left[\left(C_{5}+C_{6}\right) C_{13}^{m-\delta}+4 C_{11} K_{1}^{2} K_{2}^{m-2 \delta}\right] m^{m-\delta} .
\end{aligned}
$$

Now if we choose

$$
K_{1}:=8\left(C_{5}+C_{6}\right) \quad \text { and } \quad K_{2}:=\max \left\{C_{13}, 32 C_{11} K_{1}\right\},
$$

then we obtain (3.2) immediately. This concludes the case $\frac{n}{2} \leq q<n$.

Case 2: $n \leq q \leq \infty$. For any $p \in\left(\frac{n}{2}, n\right)$ and $n \leq q \leq \infty$, by using the Gagliardo-Nirenberg inequality (cf. [16]) we have

$$
\left\|\partial_{x}^{\beta} v(t)\right\|_{L^{q}} \leq C(n, p)\left\|\partial_{x}^{\beta} v(t)\right\|_{L^{\frac{n}{2}}}^{\theta}\left\|\partial_{x}^{2} \partial_{x}^{\beta} v(t)\right\|_{L^{p}}^{1-\theta},
$$


where $\theta$ is the unique number in $(0,1)$ determined by the following equation:

$$
-\frac{n}{q}=-2 \theta+\left(2-\frac{n}{p}\right)(1-\theta)
$$

namely,

$$
\theta= \begin{cases}\frac{2 p q+n p-n q}{(4 p-n) q} & \text { if } n \leq q<\infty \\ \frac{2 p-n}{4 p-n} & \text { if } q=\infty\end{cases}
$$

Now from (3.22), (3.23) and the result of Case 1 we see that

$$
\begin{aligned}
\left\|\partial_{x}^{\beta} v(t)\right\|_{L^{q}} \leq & C(n, p)\left(K_{1}^{-1}\left(K_{2}|\beta|\right)^{-|\beta|} t^{\frac{|\beta|}{2}}\left\|\partial_{x}^{\beta} v(t)\right\|_{L^{\frac{n}{2}}}\right)^{\theta} \\
& \times\left(K_{1}^{-1}\left(K_{2}(|\beta|+2)\right)^{-|\beta|+2} t^{\frac{|\beta|+2}{2}+1-\frac{n}{2 p}}\left\|\partial_{x}^{\beta} \partial_{x}^{2} v(t)\right\| L_{L^{p}}\right)^{1-\theta} \\
& \times K_{1}\left(K_{2}|\beta|\right)^{|\beta| \theta}\left(K_{2}(|\beta|+2)\right)^{(|\beta|+2)(1-\theta)} t^{-\frac{|\beta|}{2} \theta-\left(\frac{\beta \mid+2}{2}+1-\frac{n}{2 p}\right)(1-\theta)} \\
\leq & C(n, p) K_{1}\left(K_{2}(|\beta|+2)\right)^{|\beta|+2} t^{-\frac{|\beta|}{2}-1+\frac{n}{2 q}}
\end{aligned}
$$

It is clear that there exists a constant $C_{14} \geq 2$ such that $|\beta|^{2} \leq C_{14}^{|\beta|}$, so that

$$
\left(K_{2}(|\beta|+2)\right)^{|\beta|+2}=K_{2}^{2}|\beta|^{2}\left(1+\frac{2}{|\beta|}\right)^{|\beta|+2}\left(K_{2}|\beta|\right)^{|\beta|} \leq 9 e^{2} K_{2}^{2}\left(C_{14} K_{2}|\beta|\right)^{|\beta|} .
$$

Hence, we can choose $K_{1}$ and $K_{2}$ sufficiently large such that (3.2) holds for all $\frac{n}{2} \leq q \leq \infty$. This completes the proof of Proposition 3.1.

Having proved Proposition 3.1, we see that Theorem 1.1 immediately follows if we prove that the mild solution $(v, w)$ of $(1.1)-(1.4)$ always satisfies the regularity assumption (3.1). In what follows we prove that this is indeed the case.

Proposition 3.2. Let $\left(v_{0}, w_{0}\right) \in\left(L^{\frac{n}{2}}\left(\mathbb{R}^{n}\right)\right)^{2}$ be such that $\left\|\left(v_{0}, w_{0}\right)\right\|_{L^{\frac{n}{2}}} \leq \varepsilon$, where $\varepsilon$ is as before. Then the unique mild solution $(v, w)$ of $(1.1)-(1.4)$ satisfies (3.3) and

$$
\sup _{t>0} t^{\frac{|\beta|}{2}+1-\frac{n}{2 q}}\left\|\left(\partial_{x}^{\beta} v(t), \partial_{x}^{\beta} w(t)\right)\right\|_{L^{q}} \leq \tilde{K}_{1}\left(\tilde{K}_{2}|\beta|\right)^{|\beta|-\delta}
$$

for all $\frac{n}{2} \leq q \leq \infty, t>0$ and $\beta \in \mathbb{N}_{0}^{n}$, where $\tilde{K}_{1}$ and $\tilde{K}_{2}$ are constants depending only on $n, p, \varepsilon$ and $\delta$.

Proof. Recall that the mild solution $(v(t), w(t)) \in\left(\mathcal{X}_{p}\right)^{2}$ (for any given $p \in$ $\left.\left(\frac{n}{2}, n\right)\right)$ is obtained by using the following Picard iteration procedure:

$$
\left\{\begin{array}{l}
\left(v^{0}(t), w^{0}(t)\right)=\left(e^{t \Delta} v_{0}, e^{t \Delta} w_{0}\right), \\
v^{j+1}(t)=e^{t \Delta} v_{0}-\int_{0}^{t} e^{(t-\tau) \Delta} \nabla \cdot\left[v^{j} \nabla\left((-\Delta)^{-1}\left(w^{j}-v^{i}\right)\right)\right](\tau) d \tau, \\
w^{j+1}(t)=e^{t \Delta} w_{0}+\int_{0}^{t} e^{(t-\tau) \Delta} \nabla \cdot\left[w^{j} \nabla\left((-\Delta)^{-1}\left(w^{j}-v^{j}\right)\right)\right](\tau) d \tau .
\end{array}\right.
$$

As was shown in [22], $\left\|\left(v^{j}(t), w^{j}(t)\right)\right\|_{\mathcal{X}_{p}}$ is uniformly bounded, i.e., there exists a universal constant $C$ such that for all $t>0$,

$$
\sup _{j \geq 0} \sup _{t \geq 0}\left\|\left(v^{j}(t), w^{j}(t)\right)\right\|_{L^{\frac{n}{2}}}+\sup _{j \geq 0} \sup _{t>0} t^{1-\frac{n}{2 p}}\left\|\left(v^{j}(t), w^{j}(t)\right)\right\|_{L^{p}} \leq C \varepsilon,
$$


and, furthermore, $\left\{\left(v^{j}(t), w^{j}(t)\right)\right\}_{j=0}^{\infty}$ is a Cauchy sequence in $\left(\mathcal{X}_{p}\right)^{2}$. The solution $(v(t), w(t))$ is then obtained by taking the limit:

$$
(v(t), w(t))=\lim _{j \rightarrow \infty}\left(v^{j}(t), w^{j}(t)\right) \text { in } L^{p}\left(\mathbb{R}^{n}\right) \quad \text { for all } t>0 .
$$

To prove (3.24), we fix $\beta \in \mathbb{N}_{0}^{n}$ and set $h^{j}(t)=\left\|\left(\partial_{x}^{\beta} v^{j}(t), \partial_{x}^{\beta} w^{j}(t)\right)\right\|_{L^{q}}$. Then we can argue in the same way as in the proof of Proposition 3.1 to conclude that

$$
h^{j+1}(t) \leq \hat{B}_{\eta} t^{-\frac{|\beta|}{2}-1+\frac{n}{2 q}}+C \int_{t(1-\eta)}^{t}(t-\tau)^{-\frac{n}{2 q}} \tau^{-1+\frac{n}{2 q}} h^{j}(\tau) d \tau \quad \text { for all } j \geq 0 \text { and } t>0,
$$

where $C$ is a constant depending only on $n, p$ and $\varepsilon$, and $\hat{B}_{\eta}$ is similar to $B_{\eta}$. Hence, by applying Lemma 2.5 , there exist $\tilde{K}_{1}$ and $\tilde{K}_{2}$ depending only on $n, p, \varepsilon$ and $\delta$ such that

$$
t^{\frac{|\beta|}{2}+1-\frac{n}{2 q}}\left\|\left(\partial_{x}^{\beta} v^{j}(t), \partial_{x}^{\beta} w^{j}(t)\right)\right\|_{L^{q}} \leq \tilde{K}_{1}\left(\tilde{K}_{2}|\beta|\right)^{|\beta|-\delta} \quad \text { for all } j \geq 0 \text { and } t>0 .
$$

This yields the fact that there exist $(f(t), g(t)) \in\left(L^{q}\left(\mathbb{R}^{n}\right)\right)^{2}$ and we obtain a subsequence $\left\{\left(\partial_{x}^{\beta} v^{j_{k}}(t), \partial_{x}^{\beta} w^{j_{k}}(t)\right)\right\}$ such that

$$
\lim _{j \rightarrow \infty}\left(\partial_{x}^{\beta} v^{j_{k}}(t), \partial_{x}^{\beta} w^{j_{k}}(t)\right)=(f(t), g(t)) \text { weakly in }\left(L^{q}\left(\mathbb{R}^{n}\right)\right)^{2} \quad \text { for all } t>0 .
$$

By uniqueness of the limit, we see that $\left(\partial_{x}^{\beta} v(t), \partial_{x}^{\beta} w(t)\right)=(f(t), g(t))$. Besides, from (3.26) and (3.27) it is obvious that

$$
t^{\frac{|\beta|}{2}+1-\frac{n}{2 q}}\|(f(t), g(t))\|_{L^{q}} \leq \tilde{K}_{1}\left(\tilde{K}_{2}|\beta|\right)^{|\beta|-\delta} \quad \text { for all } t>0 .
$$

Hence, we have

$$
t^{\frac{|\beta|}{2}+1-\frac{n}{2 q}}\left\|\left(\partial_{x}^{\beta} v(t), \partial_{x}^{\beta} w(t)\right)\right\|_{L^{q}} \leq \tilde{K}_{1}\left(\tilde{K}_{2}|\beta|\right)^{|\beta|-\delta} \quad \text { for all } t>0,
$$

i.e., (3.25) holds. This completes the proof of Proposition 3.2.

Proof of Theorem 1.1. This follows immediately from Propositions 3.1 and 3.2.

\section{Proof of Theorem 1.2}

In this section we give the proof of Theorem 1.2. Similarly as in the proof of Theorem 1.1, this will be fulfilled by establishment of two propositions.

Proposition 4.1. Let the assumptions of Theorem 1.2 be satisfied. Furthermore, we assume that

$$
\left(\partial_{x}^{\beta} v(t), \partial_{x}^{\beta} w(t)\right) \in\left(C\left((0, \infty), L^{q}\left(\mathbb{R}^{n}\right)\right)\right)^{2}
$$

for all $p \leq q \leq \infty$ and $\beta \in \mathbb{N}_{0}^{n}$. Then for any $\delta \in\left(\frac{1}{2}, 1\right]$, there exist positive constants $K_{1}$ and $K_{2}$ depending only on $n, p, \varepsilon$ and $\delta$ such that

$$
\left\|\left(\partial_{x}^{\beta} v(t), \partial_{x}^{\beta} w(t)\right)\right\|_{L^{q}} \leq K_{1}\left(K_{2}|\beta|\right)^{|\beta|-\delta} t^{-\frac{|\beta|}{2}-1+\frac{n}{2 q}}
$$

for all $p \leq q \leq \infty, t>0$ and $\beta \in \mathbb{N}_{0}^{n}$. 
Proof. We prove this proposition by induction on $m=|\beta|$.

Step 1. We first assume that $m=0$. By [11], for any $\left(v_{0}, w_{0}\right) \in$ $\left(\dot{B}_{p, \infty}^{-2+\frac{n}{p}}\left(\mathbb{R}^{n}\right)\right)^{2}$ such that $\left\|\left(v_{0}, w_{0}\right)\right\|_{\dot{B}_{p, \infty}^{-2+\frac{n}{p}}} \leq \varepsilon$, the corresponding mild solution $(v(t), w(t)) \in\left(\mathcal{Y}_{p}\right)^{2}$ satisfies $\|(v(t), w(t))\|_{\mathcal{Y}_{p}} \leq C \varepsilon$ for some universal constant $C$, i.e.,

$$
\sup _{t \geq 0}\|(v(t), w(t))\|_{\dot{B}_{p, \infty}^{-2+\frac{n}{p}}}+\sup _{t>0} t^{1-\frac{n}{2 p}}\|(v(t), w(t))\|_{L^{p}} \leq C \varepsilon .
$$

This implies that (4.2) holds for $q=p$. Hence, by interpolation, it suffices to prove that $(4.2)$ also holds for $q=\infty$. Let $\eta \in(0,1)$ be a constant to be specified later. We rewrite (3.4) for $q=\infty$ as follows:

$$
\begin{aligned}
\|v(t)\|_{L^{\infty}} \leq & \left\|e^{t \Delta} v_{0}\right\|_{L^{\infty}}+\left(\int_{0}^{t(1-\eta)}+\int_{t(1-\eta)}^{t}\right) \\
& \times\left\|e^{(t-\tau) \Delta} \nabla \cdot\left[v \nabla\left((-\Delta)^{-1}(w-v)\right)\right]\right\|_{L^{\infty}}(\tau) d \tau \\
= & E_{1}+E_{2}+E_{3} .
\end{aligned}
$$

For $E_{1}$, by applying Lemma 2.2 (ii) we get

$$
E_{1} \leq \tilde{C}_{1}(n) t^{-1}\left\|v_{0}\right\|_{\dot{B}_{p, \infty}^{-2+\frac{n}{p}}} \leq \tilde{C}_{1}(n, \varepsilon) t^{-1} .
$$

For $E_{2}$, using Lemmas 2.2 (i), 2.3 and (4.3) we have

$$
\begin{aligned}
E_{2} & =\int_{0}^{t(1-\eta)}\left\|e^{(t-\tau) \Delta} \nabla \cdot\left[v \nabla\left((-\Delta)^{-1}(w-v)\right)\right]\right\|_{L^{\infty}}(\tau) d \tau \\
& \leq \tilde{C}_{2}(n, p) \int_{0}^{t(1-\eta)}(t-\tau)^{-\frac{n}{p}}\|v(\tau)\|_{L^{p}}\|(v(\tau), w(\tau))\|_{L^{p}} d \tau \\
& \leq \tilde{C}_{2}(n, p) \varepsilon^{2} \int_{0}^{t(1-\eta)}(t-\tau)^{-\frac{n}{p}} \tau^{-2+\frac{n}{p}} d \tau \\
& \leq \tilde{C}_{2}(n, p, \varepsilon) \eta^{-2} t^{-1} .
\end{aligned}
$$

For $E_{3}$, using Lemmas 2.2 (i), 2.3 and (4.3) again we get

$$
\begin{aligned}
E_{3} & =\int_{t(1-\eta)}^{t}\left\|e^{(t-\tau) \Delta} \nabla \cdot\left[v \nabla\left((-\Delta)^{-1}(w-v)\right)\right]\right\|_{L^{\infty}}(\tau) d \tau \\
& \leq \tilde{C}_{3}(n, p) \int_{t(1-\eta)}^{t}(t-\tau)^{-\frac{n}{2 p}}\|v(\tau)\|_{L^{\infty}}\|(v(\tau), w(\tau))\|_{L^{p}} d \tau \\
& \leq \tilde{C}_{3}(n, p, \varepsilon) \int_{t(1-\eta)}^{t}(t-\tau)^{-\frac{n}{2 p}} \tau^{-1+\frac{n}{2 p}}\|v(\tau)\|_{L^{\infty}} d \tau .
\end{aligned}
$$
have

Combining (4.5)-(4.7), and denoting $\tilde{B}_{\eta}=\tilde{C}_{1}(n, \varepsilon)+\tilde{C}_{2}(n, p, \varepsilon) \eta^{-2}$, we $\|v(t)\|_{L^{\infty}} \leq \tilde{B}_{\eta} t^{-1}+\tilde{C}_{3}(n, p, \varepsilon) \int_{t(1-\eta)}^{t}(t-\tau)^{-\frac{n}{2 p}} \tau^{-1+\frac{n}{2 p}}\|v(\tau)\|_{L^{\infty}} d \tau$. 
The estimate for $w(t)$ is similar. Hence,

$\|(v(t), w(t))\|_{L^{\infty}} \leq B_{\eta} t^{-1}+\tilde{C}_{4} \int_{t(1-\eta)}^{t}(t-\tau)^{-\frac{n}{2 p}} \tau^{-1+\frac{n}{2 p}}\|(v(\tau), w(\tau))\|_{L^{\infty}} d \tau$,

where $B_{\eta}=2 \tilde{B}_{\eta}$ and $\tilde{C}_{4}=2 \tilde{C}_{3}(n, p, \varepsilon)$. Now from (4.9) and Lemma 2.5 we see that (4.2) holds with $K_{1}=2 B_{\eta_{0}}$ for $m=0$ and some $\eta_{0}=\eta_{0}(n, p, \varepsilon) \in(0,1)$.

Step 2. Next we assume that $m \geq 1$ and proceed to prove that (4.2) holds for $|\beta|=m$, provided it holds for all $\beta \in \mathbb{N}_{0}^{n}$ such that $|\beta| \leq m-1$. We only need to consider the case $p \leq q<n$, because if this case is proved then the rest case $n \leq q \leq \infty$ can be proved similarly as in the corresponding case treated in Sect. 3. For this purpose, by taking the $L^{q}$-norm of $\partial_{x}^{\beta} v$, we see that for some $\eta \in(0,1)$ to be specified later, we have

$$
\begin{aligned}
\left\|\partial_{x}^{\beta} v(t)\right\|_{L^{q}} \leq & \left\|\partial_{x}^{\beta} e^{t \Delta} v_{0}\right\|_{L^{q}}+\left(\int_{0}^{t(1-\eta)}+\int_{t(1-\eta)}^{t}\right) \\
& \times\left\|\partial_{x}^{\beta} e^{(t-\tau) \Delta} \nabla \cdot\left[v \nabla\left((-\Delta)^{-1}(w-v)\right)\right]\right\|_{L^{q}}(\tau) d \tau \\
= & F_{1}+F_{2}+F_{3} .
\end{aligned}
$$

For $F_{1}$, using Lemma 2.2 (ii), it can be easily estimated that

$$
F_{1} \leq \tilde{C}_{5}(n) m^{\frac{m}{2}} t^{-\frac{m}{2}-1+\frac{n}{2 q}}\left\|v_{0}\right\|_{\dot{B}_{p, \infty}^{-2+\frac{n}{p}}} \leq \tilde{C}_{5}(n, \varepsilon) m^{m-\delta} t^{-\frac{m}{2}-1+\frac{n}{2 q}} .
$$

For $F_{2}$, similar to the derivation of (3.13), we get

$$
F_{2} \leq \tilde{C}_{6}(n, p, \varepsilon)(2 m)^{\frac{m}{2}} \eta^{-\frac{m}{2}-\frac{3}{2}} t^{-\frac{m}{2}-1+\frac{n}{2 q}} .
$$

For $F_{3}$, similar as we have done for $B_{3}$, we can obtain that

$$
\begin{aligned}
F_{3} \leq & \tilde{C}_{7}(n, p, \delta) K_{1}^{2} K_{2}^{m-2 \delta} m^{m-\delta} I(\eta) t^{-\frac{m}{2}-1+\frac{n}{2 q}} \\
& +\tilde{C}_{8}(n, p, \varepsilon) \int_{t(1-\eta)}^{t}(t-\tau)^{-\frac{n}{2 p}} \tau^{-1+\frac{n}{2 p}}\left\|\left(\partial_{x}^{\beta} v(\tau), \partial_{x}^{\beta} w(\tau)\right)\right\|_{L^{q}} d \tau,
\end{aligned}
$$

where $I(\eta)=\int_{1-\eta}^{1}(1-\tau)^{-\frac{n}{2 p}} \tau^{-\frac{m}{2}-2+\frac{n}{2 p}+\frac{n}{2 q}} d \tau$. Combining estimates (4.11)(4.13) and denoting $\tilde{B}_{\eta}$ by

$\tilde{B}_{\eta}=\tilde{C}_{5}(n, \varepsilon) m^{m-\delta}+\tilde{C}_{6}(n, p, \varepsilon)(2 m)^{\frac{m}{2}} \eta^{-\frac{m}{2}-\frac{3}{2}}+\tilde{C}_{7}(n, p, \delta) K_{1}^{2} K_{2}^{m-2 \delta} m^{m-\delta} I(\eta)$,

(4.10) implies that

$$
\begin{aligned}
\left\|\partial_{x}^{\beta} v(t)\right\|_{L^{q}} \leq & \tilde{B}_{\eta} t^{-\frac{m}{2}-1+\frac{n}{2 q}} \\
& +\tilde{C}_{8}(n, p, \varepsilon) \int_{t(1-\eta)}^{t}(t-\tau)^{-\frac{n}{2 p}} \tau^{-1+\frac{n}{2 p}}\left\|\left(\partial_{x}^{\beta} v(\tau), \partial_{x}^{\beta} w(\tau)\right)\right\|_{L^{q}} d \tau .
\end{aligned}
$$


It can be done analogously for $\partial_{x}^{\beta} w(t)$. Hence,

$$
\begin{aligned}
\left\|\left(\partial_{x}^{\beta} v(t), \partial_{x}^{\beta} w(t)\right)\right\|_{L^{q}} \leq & B_{\eta} t^{-\frac{m}{2}-1+\frac{n}{2 q}} \\
& +\tilde{C}_{9} \int_{t(1-\eta)}^{t}(t-\tau)^{-\frac{n}{2 p}} \tau^{-1+\frac{n}{2 p}}\left\|\left(\partial_{x}^{\beta} v(\tau), \partial_{x}^{\beta} w(\tau)\right)\right\|_{L^{q}} d \tau .
\end{aligned}
$$

Here $B_{\eta}=2 \tilde{B}_{\eta}$ and $\tilde{C}_{9}=2 \tilde{C}_{8}(n, p, \varepsilon)$. Having obtained (4.15), we can now follow a similar argument as that in the proof of Proposition 3.1 to get the desired assertion. We omit it here. This proves Proposition 4.1.

Proposition 4.2. Let $p \in\left(\frac{n}{2}, n\right)$. Assume that $\left(v_{0}, w_{0}\right) \in\left(\dot{B}_{p, \infty}^{-2+\frac{n}{p}}\left(\mathbb{R}^{n}\right)\right)^{2}$ be such that $\left\|\left(v_{0}, w_{0}\right)\right\|_{\dot{B}_{p, \infty}^{-2+\frac{n}{p}}} \leq \varepsilon$, where $\varepsilon$ is as before. Then the unique mild solution $(v, w)$ of (1.1)-(1.4) satisfies (4.3) and

$$
\sup _{t>0} t^{\frac{|\beta|}{2}+1-\frac{n}{2 q}}\left\|\left(\partial_{x}^{\beta} v(t), \partial_{x}^{\beta} w(t)\right)\right\|_{L^{q}} \leq \tilde{K}_{1}\left(\tilde{K}_{2}|\beta|\right)^{|\beta|-\delta}
$$

for all $p \leq q \leq \infty, t>0$ and $\beta \in \mathbb{N}_{0}^{n}$, where $\tilde{K}_{1}$ and $\tilde{K}_{2}$ are constants depending only on $n, p, \varepsilon$ and $\delta$.

Proof. The prove is essentially the same with that of Proposition 3.2, so we omit it.

Proof of Theorem 1.2. This is an immediate consequence of Propositions 4.1 and 4.2 .

\section{Acknowledgments}

This work is supported by the China National Natural Science Foundation under the Grant No. 10771223. The authors are glad to acknowledge their sincere thanks to the anonymous referees for valuable suggestions on improving the writing of this paper

\section{References}

[1] Ben Abdallah, N., Méhats, F., Vauchelet, N.: A note on the long time behavior for the drift-diffusion-Poisson system. C. R. Math. Acad. Sci. Paris 339(10), 683688 (2004)

[2] Biler, P., Dolbeault, J.: Long time behavior of solutions to Nernst-Planck and Debye-Hückel drift-diffusion systems. Ann. Henri Poincaré 1, 461-472 (2000)

[3] Biler, P., Hebisch, W., Nadzieja, T.: The Debye system: existence and large time behavior of solutions. Nonlinear Anal. 23, 1189-1209 (1994)

[4] Corrias, L., Perthame, B., Zaag, H.: Global solutions of some chemotaxis and angiogenesis systems in high space dimensions. Milan J. Math. 72, 1-28 (2004) 
[5] Debye, P., Hückel, E.: Zur Theorie der Elektrolyte, II: Das Grenzgesetz für die elektrische Leitfähigkeit. Phys. Z. 24, 305-325 (1923)

[6] Gajewski, H., Gröger, K.: On the basic equations for carrier transport in semiconductors. J. Math. Anal. Appl. 113, 12-35 (1986)

[7] Gogny, D., Lions, P.-L.: Sur les états d'équilibre pour les densités électroniques dans les plasmas. RAIRO Modél. Math. Anal. Numér. 23, 137-153 (1989)

[8] Giga, Y., Sawada, O.: On regularizing-decay rate estimates for solutions to the Navier-Stokes initial value problem. Nonlinear Anal. 1(2), 549-562 (2003)

[9] Jerome, J.W.: Analytical approaches to charge transport in a moving medium. Transp. Theory Stat. Phys. 31, 333-366 (2002)

[10] Kahane, C.: On the spatial analyticity of solutions of the Navier-Stokes equations. Arch. Ration. Mech. Anal. 33, 386-405 (1969)

[11] Karch, G.: Scaling in nonlinear parabolic equations. J. Math. Anal. Appl. 234, 534-558 (1999)

[12] Karch, G.: Scaling in nonlinear parabolic equations. Applications to Debye system. AIP Conf. Proc. 553(1), 243-248 (2001)

[13] Kurokiba, M., Ogawa, T.: Well-posedness for the drift-diffusion system in $L^{p}$ arising from the semiconductor device simulation. J. Math. Anal. Appl. 342(2), 1052-1067 (2008)

[14] Lemarié-Rieusset, P.-G.: Recent developments in the Navier-Stokes problem. In: Research Notes in Mathematics. Chapman \& Hall/CRC, London (2002)

[15] Miura, H., Sawada, O.: On the regularizing rate estimates of Koch-Tataru's solution to the Navier-Stokes equations. Asymptot. Anal. 49, 1-15 (2006)

[16] Nirenberg, L.: On elliptic partial differential equations. Ann. Sc. Norm. Sup. Pisa. Ser. III 13, 115-162 (1959)

[17] Ogawa, T., Shimizu, S.: The drift-diffusion system in two-dimensional critical Hardy space. J. Funct. Anal. 255(5), 1107-1138 (2008)

[18] Ogawa, T., Yamamoto, M.: Asymptotic behavior of solutions to driftdiffusion system with generalized dissipation. Math. Models Methods Appl. Sci. 19(6), 939-967 (2009)

[19] Sawada, O.: On analyticity rate estimates of the solutions to the Navier-Stokes equations in Bessel-potential spaces. J. Math. Anal. Appl. 312, 1-13 (2005)

[20] Selberherr, S.: Analysis and Simulation of Semiconductor Devices. Springer, Berlin (1983)

[21] Zhao, J., Cui, S.: Remarks on the local existence of solutions to the Debye system. J. Math. Anal. Appl. (2011). doi:10.1016/j.jmaa.2011.05.027

[22] Zhao, J., Deng, C., Cui, S.: Well-posedness of a dissipative system modeling electrohydrodynamics in Lebesgue spaces (2010, preprint) 
Jihong Zhao, Qiao Liu and Shangbin Cui

Department of Mathematics

Sun Yat-sen University

Guangzhou

510275 Guangdong

People's Republic of China

e-mail: zhaojihong2007@yahoo.com.cn

Qiao Liu

e-mail: liuqao2005@163.com

Shangbin Cui

e-mail: cuisb3@yahoo.com.cn

Received: 19 August 2010.

Accepted: 5 May 2011. 